\title{
Aqueous Nanofluid as a Two-Phase Coolant for PWR
}

\author{
Pavel N. Alekseev, Yury M. Semchenkov, and Alexander L. Shimkevich
}

NRC "Kurchatov Institute", 1 Kurchatov Square, Moscow 123182, Russia

Correspondence should be addressed to Alexander L. Shimkevich, shall@dhtp.kiae.ru

Received 19 May 2012; Revised 28 August 2012; Accepted 11 September 2012

Academic Editor: Boštjan Končar

Copyright ( 2012 Pavel N. Alekseev et al. This is an open access article distributed under the Creative Commons Attribution License, which permits unrestricted use, distribution, and reproduction in any medium, provided the original work is properly cited.

\begin{abstract}
Density fluctuations in liquid water consist of two topological kinds of instant molecular clusters. The dense ones have helical hydrogen bonds and the nondense ones are tetrahedral clusters with ice-like hydrogen bonds of water molecules. Helical ordering of protons in the dense water clusters can participate in coherent vibrations. The ramified interface of such incompatible structural elements induces clustering impurities in any aqueous solution. These additives can enhance a heat transfer of water as a two-phase coolant for PWR due to natural forming of nanoparticles with a thermal conductivity higher than water. The aqueous nanofluid as a new condensed matter has a great potential for cooling applications. It is a mixture of liquid water and dispersed phase of extremely fine quasi-solid particles usually less than $50 \mathrm{~nm}$ in size with the high thermal conductivity. An alternative approach is the formation of gaseous (oxygen or hydrogen) nanoparticles in density fluctuations of water. It is possible to obtain stable nanobubbles that can considerably exceed the molecular solubility of oxygen (hydrogen) in water. Such a nanofluid can convert the liquid water in the nonstoichiometric state and change its reduction-oxidation (RedOx) potential similarly to adding oxidants (or antioxidants) for applying 2D water chemistry to aqueous coolant.
\end{abstract}

\section{Introduction}

It is well known [1] that the microstructure of liquid water is not understood, and its dynamic hydrogen-bonds ( $\mathrm{HB}$ ) structure has been the subject of intense debate for decades. Ice, whose $\mathrm{HB}$ structure was long ago well established, forms a tight "tetrahedral" lattice of molecules each binding to four others. The prevailing model of liquid water holds that as ice melts, the molecules loosen their grip but remain generally arranged in the same tetrahedral groups. This hydrogenbonding pattern has been assumed to account for water properties.

However the majority of molecules were found in higher density regions with an asymmetric disordered structure where some islands of tetrahedral order were floated [2]. The greater density of liquid water in these regions implies that the molecules are more closely packed there than the simple tetrahedrons seen in ice $[3,4]$.

The conclusion $[2,5]$ that a dominant fraction of the molecules in liquid water are very asymmetrically hydrogen bonded with only two well-defined H-bonds (one donating and one accepting) is in strong contrast to the accepted picture as being near tetrahedral, $\mathrm{H}$ bonded. From small-angle $\mathrm{X}$-ray scattering studies, they furthermore find evidence for density nonhomogeneity on a length-scale of $1 \mathrm{~nm}$ indicating that the two components are spatially separated on the time scale of the experiment. The recent controversy about the structure of liquid water pits new models involving water molecules in relatively stable rings-and-chains structures against the standard model that posits water molecules in slightly distorted tetrahedral coordination. The current study is giving new life to familiar Rontgen's "two-structure" model of liquid water $[3,4]$.

A topological structure of density fluctuations in condensed matter has been studied by molecular-dynamics (MD) simulation as ramified clusters of almost regular Delaunay's simplexes (tetrahedrons) built on the fours of densely packed atoms and connected in pairs by faces as tetrahedral Bernal's chains $[6,7]$. The review of publications on this subject is presented in the monograph [8] and a topological criterion [9-11] is offered for finding these simplexes exactly. Such a criterion allows making the selection of 
the dense-part simplexes by fixing the overall length of their edges in a point of maximum number of obtained clusters in the MD cell.

At the same time, any nanofluid as a new coolant is a suspension of nanoparticles with sizes less than $100 \mathrm{~nm}$ and volume fractions typically less than 4\% [12]. Such a coolant has shown the ability of enhancement (up to 40\%) in thermal conductivity compared with the base liquid [13] and a significant increase of critical heat flux [14]. Oxides $\left(\mathrm{Al}_{2} \mathrm{O}_{3}, \mathrm{CuO}, \mathrm{TiO}_{2}\right)$, nitrides (AlN, $\left.\mathrm{SiN}\right)$, carbides ( $\left.\mathrm{SiC}, \mathrm{TiC}\right)$, and metals $(\mathrm{Ag}, \mathrm{Au}, \mathrm{Cu}, \mathrm{Fe})$ can be used in the nanofluid as nanoparticle materials [15].

Presently, nanofluids are produced by two techniques [15]. A two-step technique starts with nanoparticles produced by one of physical or chemical synthesis techniques as a dry powder and then dispersed into the base liquid. This method may result in a large degree of nanoparticle agglomeration. The single-step (evaporation) one simultaneously makes and disperses the nanoparticles directly into the base liquid. The two-step process produces nanofluids with oxide nanoparticles and the single-step one produces the nanofluids with metal nanoparticles.

They are unlikely to become the mainstay of commercial nanofluid production due to the required low vapour pressure (typically less than $10 \mathrm{~Pa}$ ) that limits the rate of nanofluid production and makes it expensive. Although nanoparticle agglomeration in this case is minimized as a result of the liquid flowing continuously, the effect of temperature and operation conditions on allocation of nanoparticles may be significant due to changing the electric potential on the surface of colloidal particles as a main factor to provide the stability of nanofluid [16].

Therefore systematic studying of the aqueous nanofluids is needed [17-20] since a key factor in understanding their thermal properties is the clustering effects that provide paths for rapid heat transport and stabilize nanofluid composition in different conditions.

\section{Some Aspects of Liquid Water Microstructure}

Precise experimental techniques for determining the local structure of liquid water are missing since each water molecule undergoes rapid rearrangement on the order of femtoseconds. The need for a better understanding of water at the microscopic level has forced the development of computational methods that describe the individual and cooperative structure and dynamics of water molecules, and many studies have been carried out using these techniques. These simulations predict locally ordered hydrogen-bonding clusters of water molecules that continually form and break [21].

MD simulations of density fluctuations in liquid metals $[9,10]$ have shown that their dense tetrahedral clusters are characterized by vertex connections as well as in liquid water the low-density ice crystallites are divided by dense tetrahedral clusters with an asymmetrical structure [1]. However these clusters (see Figure 1(a)) are more complicated due to the effect of hydrogen bonds but the frame of them as a broken red line connecting the centers of tetrahedrons is also ramified [20].

One can expect that water molecules have enough time for rebuilding hydrogen bonds in a dense part of water density fluctuations due to very fast librations and rotations of them in liquid phase. From this, the model for instant dense clusters [22] is built with helices of hydrogen bonds (see Figure 1(b)). All angles between them are equal to $106.8^{\circ}$. Each water molecule in such clusters is tetrahedrally bonded with three molecules of the same cluster and with one of some ice crystallites in liquid water.

The topology of helical clusters is essentially differed from the one of the crystalline ice. From this and only this point of view, the liquid water is considered as a twostructural fluid by dynamic forming the two topological kinds of clusters in density fluctuations. The mole fraction of helical clusters in liquid water can amount to 0.6 [22].

The dense helical clusters save, in principle, four hydrogen bonds for each molecule of water; three of them are internal and one is external for connecting to ice crystallites. They can stimulate coherent proton vibrations in the coil of helical cluster if these protons are ordered in the helix (see Figure 1(b)). A spectral series, $v(n)$, of coherent proton vibrations in the helical cluster as a function of watermolecules number, $n$, is [22]

$$
v[\mathrm{THz}] \sim 22 \frac{\sqrt{n-1}}{n}, \quad \text { at } n \geq 6
$$

A possible generation of coherent proton vibrations by an external electromagnetic impact at these resonance frequencies can selectively amplify each mode and, thus, can strengthen water microheterogeneity far from its thermodynamic equilibrium. Therefore studying the electromagnetic absorption at these frequencies can be interesting both for revealing spectral lines (1), and for creating a possible technique for managing the microstructure of water. Moreover, additives in aqueous solution can be concentrated in the ramified interface of two dynamic microstructures of liquid water that can cause fluctuation-induced clustering of the impurities in nanoparticles and thus, forming a stable twophase state of aqueous solution $[20,23]$.

\section{Clustering Impurities in Liquid Water}

A spatial position of any impurity in liquid solution has dual character due to density fluctuations of the solvent that build instant dense clusters of almost regular tetrahedrons connected in pairs by faces in ramified $n$-chains from solvent atoms $[6,11]$. At a low concentration of impurity, its atoms place on the external faces of the dense solvent clusters. In increasing concentration, the impurity atoms build their own dense tetrahedral clusters in the solution so that it becomes microheterogeneous as a nanofluid [23].

In liquid water, tetrahedral clusters of water density fluctuations (see Figure 1(b)) are more complicated than the ones in simple liquids due to hydrogen bonds but the frame of them as a broken red line (see Figure 1(a)) connecting the centres of tetrahedrons is also ramified [19]. 


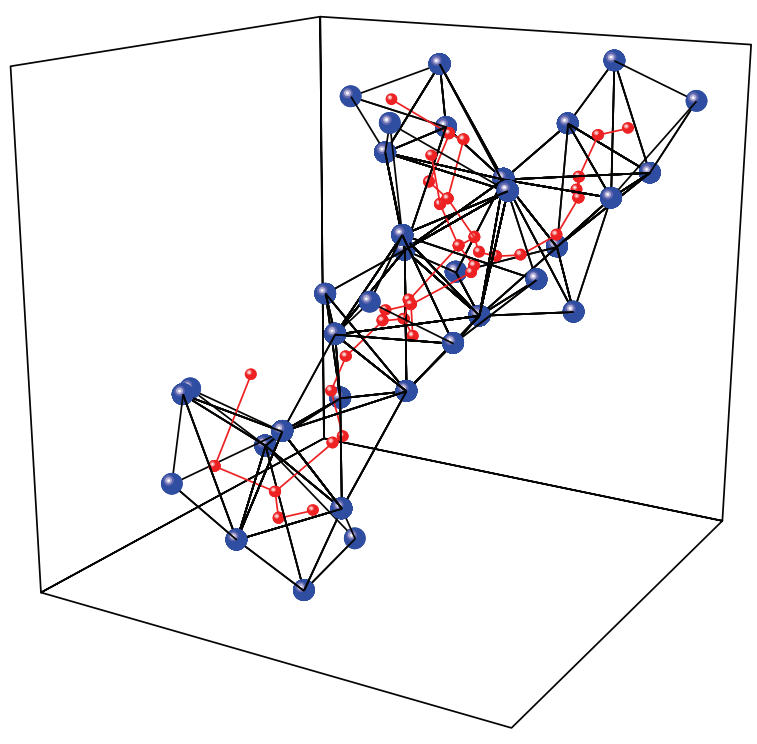

(a)

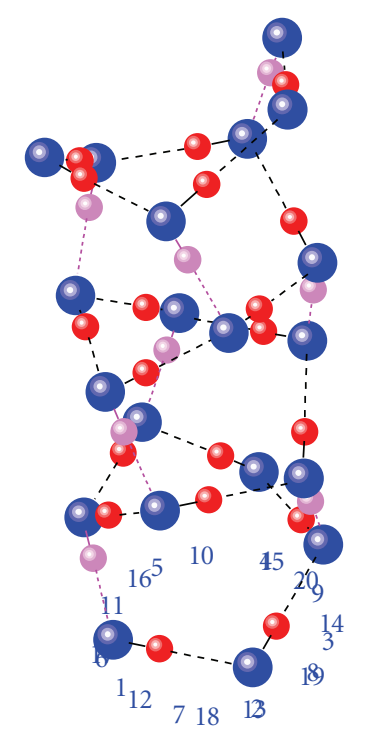

(b)

Figure 1: (a) A dense tetrahedral cluster in MD model of liquid water at $300 \mathrm{~K}$ and its frame (broken red line); blue points are the molecules and red points are centres of the cluster tetrahedrons; (b) the helical atomic model for this cluster with possible coherent exchange of protons (red balls) between oxygen atoms (blue balls) along the helix of hydrogen bonds (black lines) and hydrogen-bond bridges (pink balls); the projections of oxygen atoms in the plane are denoted by numbers.

Thus, the impurities residence in liquid water is practised in the interface of low- and high-density regions. At some concentration of impurity atoms, they form their own dense tetrahedral clusters in the aqueous solution. Such clusters ("inherent" nanoparticles) will be stable at different conditions of the water-coolant operation [20].

In this connection, it is offered a fractal model for inherent nanoparticles that can appear in the water coolant under some conditions as percolation clusters of solid-like

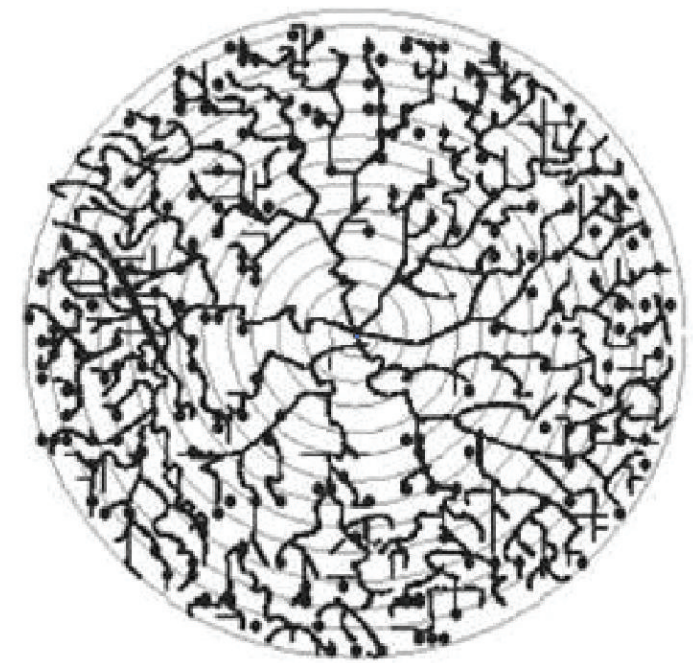

Figure 2: The scheme of percolation fractal cluster of solid-like filaments.

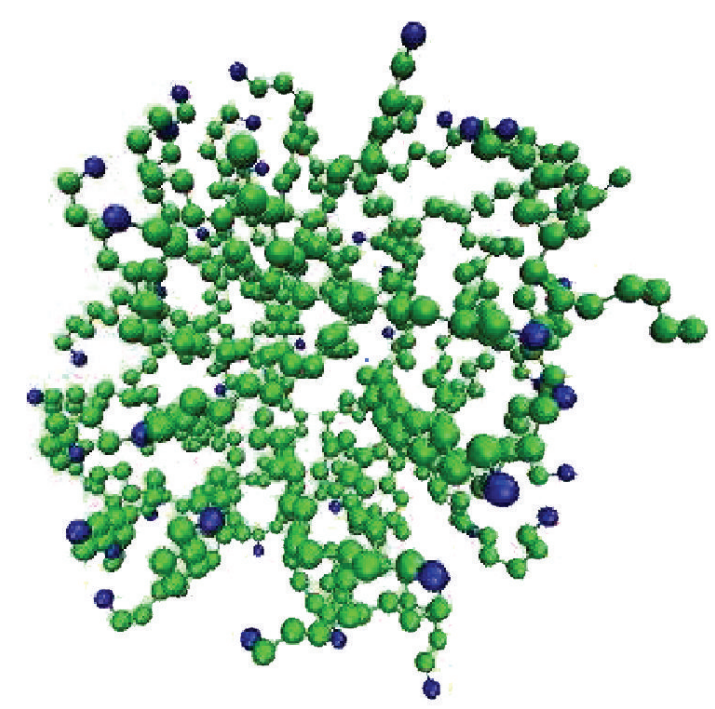

Figure 3: The fractal cluster as an inherent micelle in liquid water.

filaments [19] shown in Figure 2. Such a fractal nanoparticle is a micelle presented in Figure 3.

In [24], it is shown that fractal matter, $M$, enclosed in a sphere of radius, $r$, satisfies the scaling law

$$
M(r) \sim r^{D},
$$

where $D$ is Hausdorff's dimension of particle fractal which is equal to $\sim 2.5$.

In any real cluster, the fractal structure observed on scales, $r$, satisfied the condition of $a<r<d / 2$, where $d$ is the size of fractal nanoparticle and $a$ is the thickness of fractal filament. In that case, one can easily obtain the volume fraction, $\varphi_{f}$, of particle material in the fractal as [19]

$$
\varphi_{f}=(3 / D)(2 a / d)^{3-D} .
$$




\section{Evaluating Heat Transfer in Liquid Water by Fractal Nanoparticles}

Thermal properties of fiber constituents (filaments) are locally anisotropic but the same properties of a clew of filaments are isotropic [19]. Therefore the thermal conductivity, $\lambda_{f}$, of fractal mater as a percolation cluster filled with liquid is the same as the solid nanoparticle with respect to heat flow in the fluid and presented as $\lambda_{f} \equiv \lambda_{m}$. Here $\lambda_{m}$ is the thermal conductivity of dispersed material.

At the same time, spherical fractal particles have developed interface of the solid/liquid contact (see Figure 2) and its heat-variable resistor is negligible. Then, we can use the potential theory of Maxwell [25] for well-dispersed fractal nanoparticles that gives a simple relationship for the thermal conductivity, $\lambda_{n}$, of nanofluid (with randomly distributed and noninteracting spherical particles) in the reduced form

$$
\frac{\lambda_{n}}{\lambda} \approx 1+3 \varphi_{p} \frac{(1-\alpha)}{(1+2 \alpha)}
$$

where $\lambda$ is the thermal conductivity of liquid matrix, $\varphi_{p}$ is the volume fraction of nanoparticles in liquid, and $\varphi \equiv \varphi_{f} \varphi_{p}$ is the volume fraction of their material in the liquid; $\alpha=$ $\lambda / \lambda_{m} \ll 1$. Since $\varphi_{p} \equiv \varphi / \varphi_{f}=(D / 3)(2 a / d)^{D-3} \varphi$ in [19], the following equation is obtained:

$$
\frac{\left(\left(\lambda_{n} / \lambda\right)-1\right)}{\varphi} \approx D\left(\frac{2 a}{d}\right)^{D}-\frac{3(1-\alpha)}{1+2 \alpha} .
$$

The function (5) of three parameters: $D, 2 a / d$, and $\alpha$ is calculated for estimating the effect of fractal structure of nanoparticles on the thermal conductivity of aqueous nanofluid that is given in Table 1 .

\section{Discussion of Results}

According to $[20,23]$, the lower limit of the impurity concentration for its clustering in liquid water is $0.1 C_{s}$, where $C_{s}$ is the saturation impurity concentration. It is clear that this range for clustering impurities dissolved in water as "solid-like" nanoparticles is an effective way to stabilize the aqueous nanofluid structure for different conditions (high temperature, flow rate, radiation, etc) of its operation in any power system. In the limit of a small volume fraction of such nanoparticles and their high thermal conductivity, the enhancement of thermal conductivity of aqueous nanofluid can be $18 \varphi$ for spherical fractal particles of $10-50 \mathrm{~nm}$ in size composed of fine filaments of $0.5-1.0 \mathrm{~nm}$ in diameter. The significant property of considered fractal structure of disperse phase in nanofluids is an explanation of observed enhancement of their kinetic characteristics obtained in different experiments.

At the same time, it is important to understand that the fractal particles are the product of complex chemical reactions between any dissolved impurity and the aqueous solvent. Therefore, it is necessary to develop a special technology for getting them and stabilizing them in liquid matter.

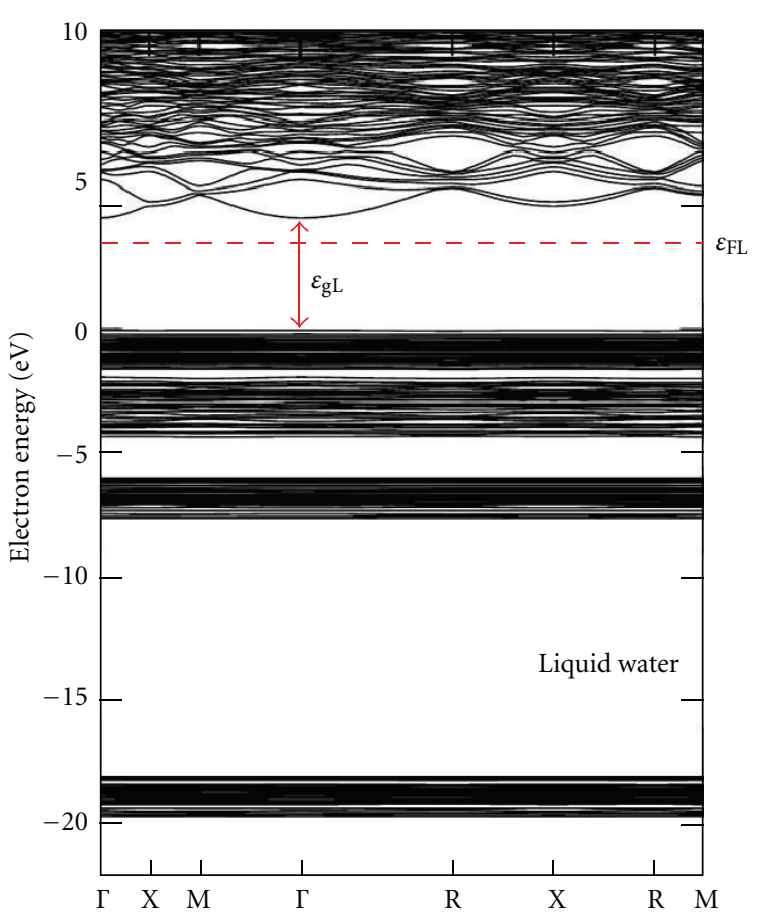

(k)

(a)

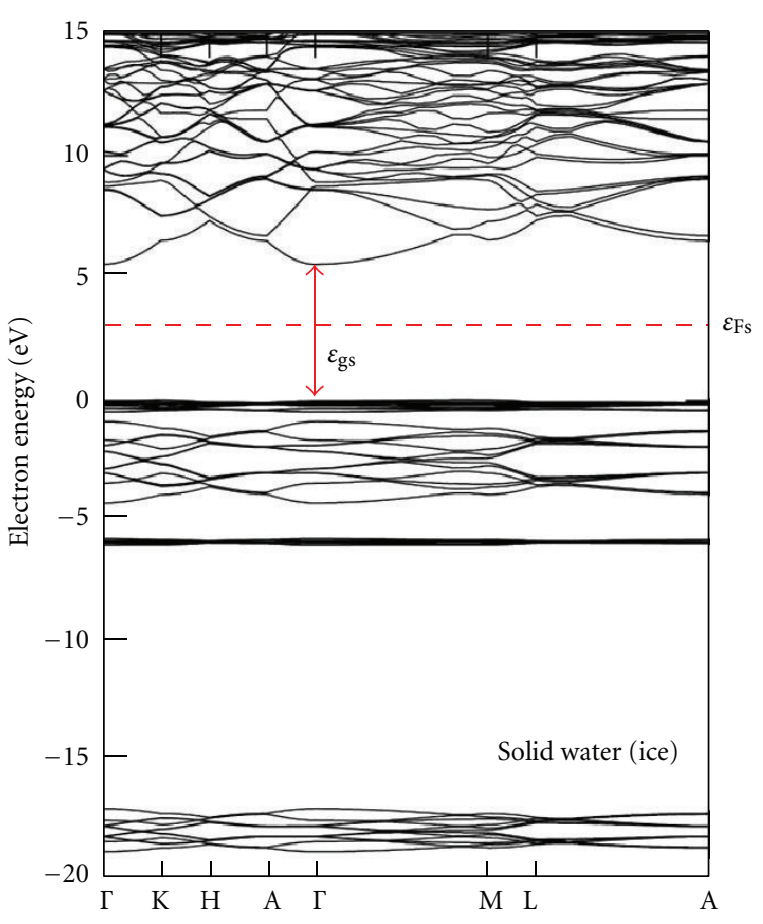

(k)

(b)

FIgure 4: Brillouin bands of liquid (a) and solid (b) water [26]; here the zero energy of electrons corresponds to the top of valence band and red dotted lines are Fermi levels, $\varepsilon_{\mathrm{Fl}}$ and $\varepsilon_{\mathrm{Fs}}$, of electrons in liquid and solid water with corresponding band gaps, $\varepsilon_{\mathrm{gl}}$ and $\varepsilon_{\text {gs }}$. 


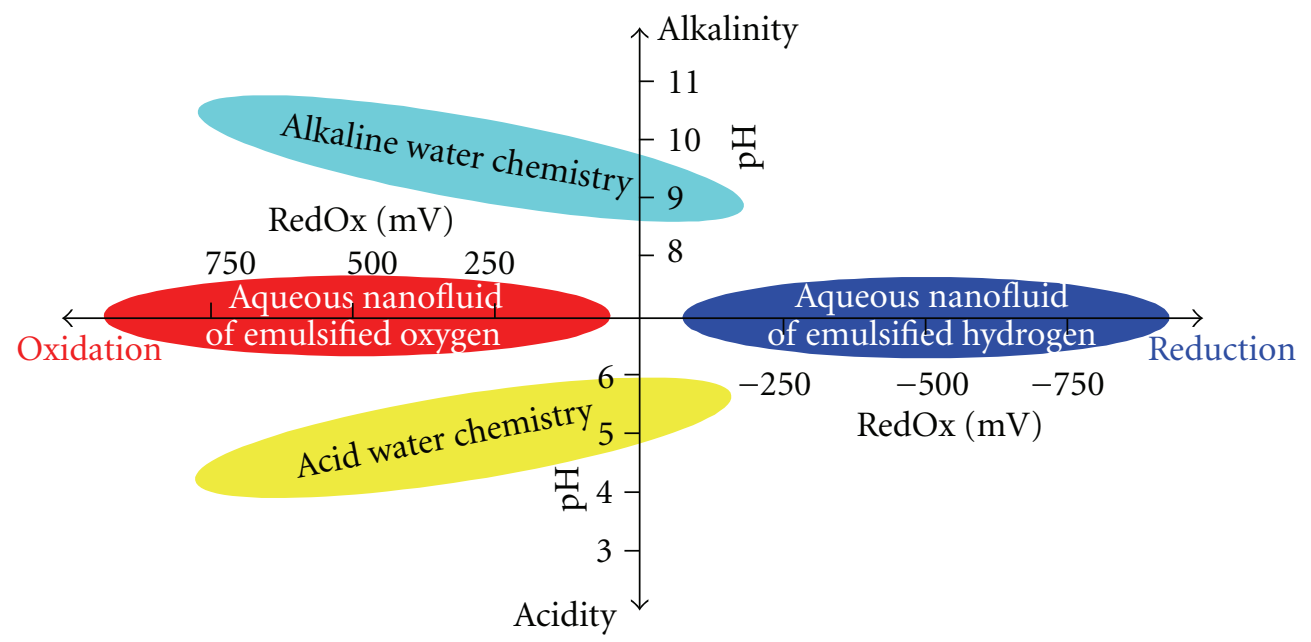

Figure 5: Diagram of aqueous chemistry as 2D plot of $\mathrm{pH}$ and RedOx parameters.

TABLE 1: The enhancement of water thermal conductivity by fractal nanoparticles.

\begin{tabular}{lcccccccccccc}
\hline & \multicolumn{3}{c}{$\mathrm{Al}_{2} \mathrm{O}_{3}$} & & & $\mathrm{Al}$ & & $\mathrm{Cu}$ & \multicolumn{3}{c}{ Carbon fiber } \\
\hline$D$ & 3.0 & 2.7 & 2.5 & 3.0 & 2.7 & 2.5 & 3.0 & 2.7 & 2.5 & 3.0 & 2.7 & 2.5 \\
$2 a / d$ & - & 0.02 & 0.1 & - & 0.02 & 0.1 & - & 0.02 & 0.1 & - & 0.02 & 0.1 \\
$\alpha$ & 0.02 & 0.02 & 0.02 & 0.002 & 0.002 & 0.002 & 0.001 & 0.001 & 0.001 & 0 & 0 & 0 \\
$\left(\lambda_{0} /(\lambda-1)\right) / \varphi$ & 2.87 & 8.35 & 7.56 & 2.98 & 8.68 & 7.86 & 2.99 & 5.37 & 17.6 & 3.0 & 8.73 & 17.7 \\
\hline
\end{tabular}

The theoretical studies [11] show that the fluctuationinduced clustering of impurities is possible only if the solid-like disperse phase (colloids) in aqueous solution is hydroxides. Therefore, it is necessary to select correctly impurities for water solution in order to get the stable nanosuspension in it [20].

In this connection, a nanofluid with gaseous nanoparticles (bubble stones) is interested especially with oxygen (hydrogen) nanobubbles. These gaseous nanoparticles are gathered in fluctuation-induced defects of tetrahedral water structure mentioned above. Obviously, such formation of gaseous nanoemulsion in aqueous solution allows obtaining comparatively stable nanobubbles that can considerably exceed the molecular solubility of gaseous impurities in liquid water at the given temperature.

Moreover, the aqueous nanofluid of emulsified oxygen (hydrogen) converts the liquid water in the nonstoichiometric state that is illustrated in Figure 4(a) by a shift of Fermi level, $\varepsilon_{\mathrm{Fl}}$, in the band gap, $\varepsilon_{\mathrm{gl}}$, of liquid water. Here is the hypostoichiometric state of water by emulsifying hydrogen in it. As seen in Figure 4(b), Fermi level, $\varepsilon_{\mathrm{Fs}}$, of solid water is in the middle of band gap, $\varepsilon_{\text {gs }}$, that is corresponded to the stoichiometric state of ice as a characteristic electron state of solid water.

It is known [27] that the change of Fermi level in the band-gap of any nonmetal liquid is equivalent to changing a Reduction-Oxidation (RedOx) potential of this melt. This can concern the aqueous nanofluid with emulsified oxygen (hydrogen) as it is shown in Figure 5 in the plane of two coordinates, $\mathrm{pH}$ and RedOx [28]. The first parameter defines the logarithmic portion of hydrogen cations in liquid water, that is, alkalinity or acidity of the aqueous matter, and the second one reflects nonstoichiometric state, $\mathrm{H}_{2} \mathrm{O}_{1 \pm x}$, of liquid water as the condensed matter, that is, the presence in it of dissolved hydrogen or oxygen.

It is visible that the aqueous chemistry accepted in power engineering of pressure-water reactor (PWR) is the oxidative one due to continuous additives of feed water that naturally dissolves oxygen in it. This impurity is not desirable for the aqueous chemistry of the PWR first-loop coolant due to strong oxidizing structural materials providing continuous growth of oxide films on the surface of fuel cladding.

At the same time, the PWR operation experience shows that this film of thickness more than $10-15$ microns is fragile and sensitive to thermal cycles. The film is locally breaking and opens the fuel cladding to corrosion by zirconium-steam reaction.

Therefore, it is important to organize an effective technological process for removing oxygen from the feed water. In hydrogen "rinsing" of this water, it is possible to convert RedOx potential of the aqueous coolant in the negative value area (see Figure 5) and obtain the corrosion-passive one that can inhibit the growth of oxide films on the surface of fuel cladding and local breakup of them.

\section{Conclusions}

The theoretical studies show how one can provide the stable formation of nanoparticles in water solution. It is important to form clusters in water directly from impurities that are dissolved there. Then, ramified fractal clusters, as natural 
solid-like part of solution, can be stable constituents of the aqueous nanofluid.

It will provide the stability of water nanofluid embedded with such nanoparticles that flow in the first loop of PWR without changing their microstructure.

\section{Acknowledgments}

The authors are pleased to acknowledge Dr. A. S. Kolokol for giving some data on molecular-dynamic simulation of water structure and discussing this work that is supported by the Russian Foundation of Basic Researches (Grant no. 10-0800217).

\section{References}

[1] C. Huanga, K. T. Wikfeldtb, T. Tokushima et al., "The inhomogeneous structure of water at ambient conditions," Proceedings of the National Academy of Sciences of the United States of America, vol. 106, no. 36, pp. 15214-15218, 2009.

[2] P. Wernet, D. Nordlund, U. Bergmann et al., "The structure of the first coordination shell in liquid water," Science, vol. 304, no. 5673, pp. 995-999, 2004.

[3] E. Cartlidge, The Strangest Liquid, New Scientist, 2010.

[4] K. Tuttle, Researchers Rediscover the Structureof Water, SLAC Today, 2010.

[5] T. Tokushima, Y. Harada, O. Takahashi et al., "High resolution $\mathrm{X}$-ray emission spectroscopy of liquid water: the observation of two structural motifs," Chemical Physics Letters, vol. 460, no. 4-6, pp. 387-400, 2008.

[6] J. D. Bernal, "The structure of liquids," Proceedings of the Royal Society A, vol. 280, p. 299, 1964.

[7] Y. Waseda, The Structure of Non-Crystalline Materials, Liquids and Amorphous Solids, McGraw-Hill, New York, NY, USA, 1980.

[8] N. N. Medvedev, Voronoy-Delaunay Method in Research of Structure of Non-Crystalline Systems Novosibirsk, Siberian Branch of the Russian Academy of Science, 2000.

[9] A. S. Kolokol and A. L. Shimkevich, "Topological structure of liquid metals," Atomic Energy, vol. 98, no. 3, pp. 187-190, 2005.

[10] A. L. Shimkevich, A. S. Kolokol, and I. Y. Shimkevich, "Twostructure model for simple metals," Journal of Non-Crystalline Solids, vol. 353, no. 32-40, pp. 3472-3474, 2007.

[11] A. S. Kolokol and A. L. Shimkevich, "Topological structure of density fluctuations in condensed matter," Journal of NonCrystalline Solids, vol. 356, no. 4-5, pp. 220-223, 2010.

[12] S. Choi, "Enhancing thermal conductivity of fluids with nanoparticles," in Development and Applications of NonNewtonian Flows, D. A. Siginer and H. P. Wang, Eds., p. 99, ASME, New York, NY, USA, 1995.

[13] M. Bahrami et al., "Assessment of relevant physical phenomena controlling thermal performance of nanofluids," in Proceedings of the ASME International Mechanical Engineering Congress and Expo (IMECE '06), Chicago, Ill, USA, November 2006.

[14] S. M. You, J. H. Kim, and K. H. Kim, "Effect of nanoparticles on critical heat flux of water in pool boiling heat transfer," Applied Physics Letters, vol. 83, no. 16, pp. 3374-3376, 2003.

[15] W. Yu et al., "Review and assessment of nanofluid technology for transportation and other applications," Report of Argonne National Laboratory ANL/ESD/07-9, 2007.

[16] Nanofluid Datasheet, "Meliorum Technologies," 2008.
[17] C. H. Lo and T. T. Tsung, "Low-than-room temperature effect on the stability of $\mathrm{CuO}$ nanofluid," Reviews on Advanced Materials Science, vol. 10, no. 1, pp. 64-68, 2005.

[18] P. Keblinski, S. R. Phillpot, S. U. S. Choi, and J. A. Eastman, "Mechanisms of heat flow in suspensions of nano-sized particles (nanofluids)," International Journal of Heat and Mass Transfer, vol. 45, no. 4, pp. 855-863, 2001.

[19] A. L. Shimkevich, "On enhancing water heat transfer by nanofluids," in Proceedings of the 17th International Conference on Nuclear Engineering (ICONE '09), pp. 19-22, July 2009.

[20] P. N. Alekseev et al., "On basic principles for modifying water as a coolant of PWR," in Proceedings of the Transactions of European Nuclear Conference (ENC '10), Barcelona, Spain, 2010.

[21] R. Roy, W. A. Tiller, I. Bell, and M. R. Hoover, "The structure of liquid water; novel insights from materials research; potential relevance to homeopathy," Materials Research Innovations, vol. 9, no. 4, pp. 98-103, 2005.

[22] A. L. Shimkevich and I. Y. Shimkevich, "On water density fluctuations with helices of hydrogen bonds," Advances in Condensed Matter Physics, vol. 2011, Article ID 871231, 5 pages, 2011.

[23] A. L. Shimkevich, The Composition Principles for Designing Nuclear-Reactor Materials, Edited by N. N. PonomarevStepnoi, IzdAt, Moscow, Russia, 2008.

[24] V. E. Tarasov, "Fractional hydrodynamic equations for fractal media," Annals of Physics, vol. 318, no. 2, pp. 286-307, 2005.

[25] J. C. Maxwell, A Treatise on Electricity and Magnetism, Dover, New York, NY, USA, 3rd edition, 1954.

[26] G. Galli, Electronic Properties of Water, University of California, http://angstrom.ucdavis.edu/.

[27] P. N. Alekseev and A. L. Shimkevich, "On voltage-sensitive managing the redox-potential of msr fuel composition," in Proceedings of the 16th International Conference on Nuclear Engineering (ICONE '08), pp. 21-29, May 2008.

[28] D. Langmuir, Aqueous Environmental Chemistry, Prentice Hall, New Jersey, NJ, USA, 1997. 

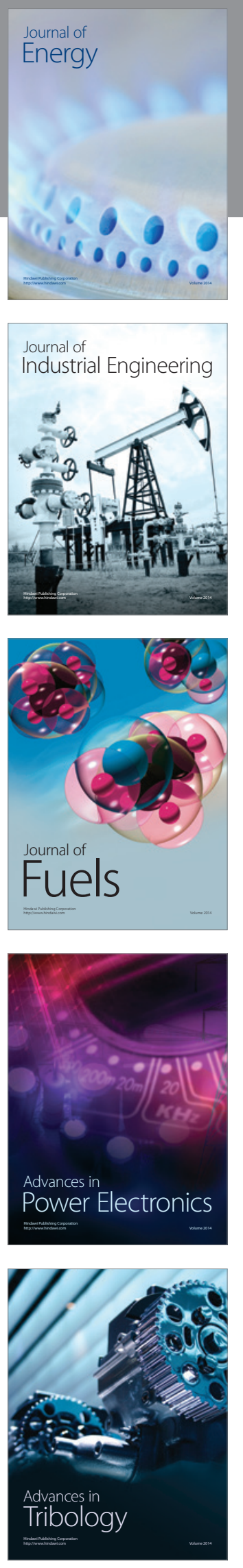
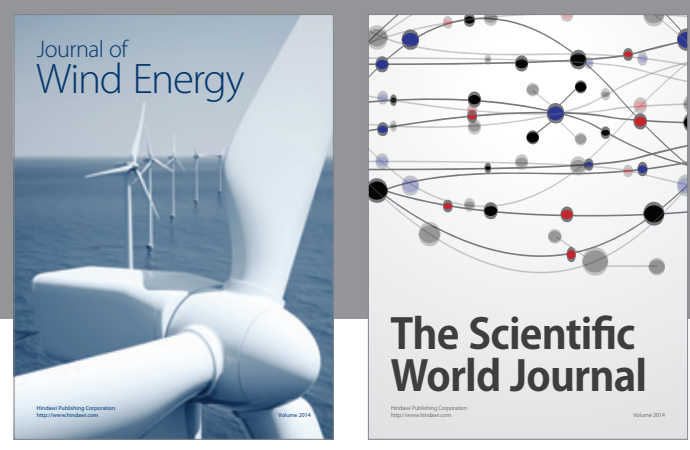

The Scientific World Journal

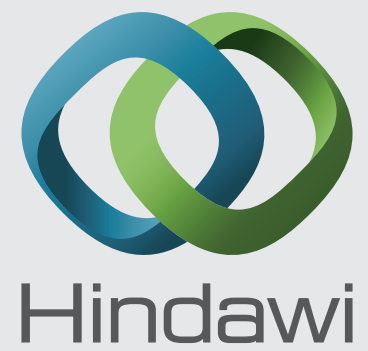

Submit your manuscripts at http://www.hindawi.com
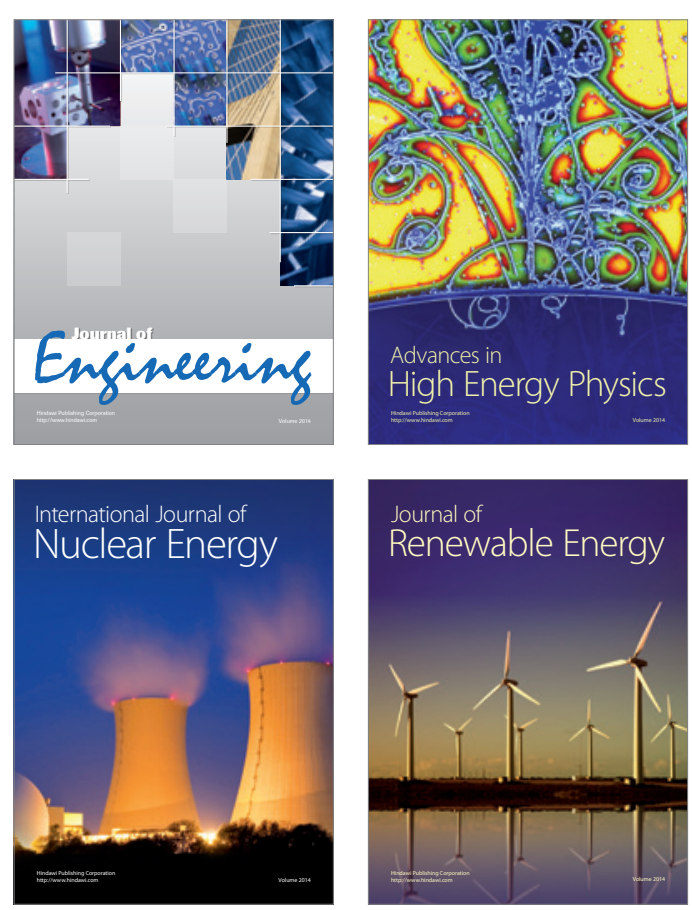

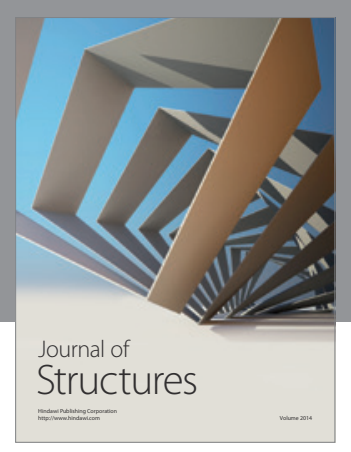

Rotating
Mechinery
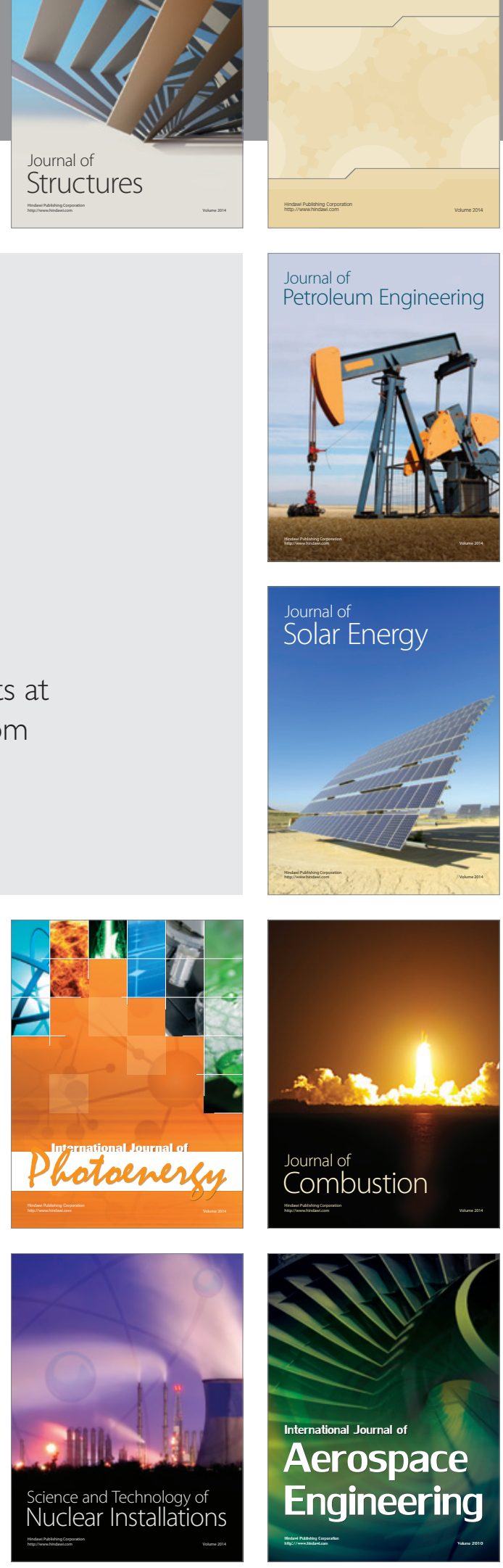